\title{
Uniqueness theorems for harmonic maps into metric spaces
}

\author{
Chikako Mese*
}

\begin{abstract}
Recent developments extend much of the known theory of classical harmonic maps between smooth Riemannian manifolds to the case when the target is a metric space of curvature bounded from above. In particular, the existence and regularity theorems for harmonic maps into these singular spaces have been successfully generalized. Furthermore, the uniqueness of harmonic maps is known when the domain has a boundary (with a smallness of image condition if the target curvature is bounded from above by a positive number). In this paper, we will address the question of uniqueness when the domain space is without a boundary in two cases: one, when the curvature of the target is strictly negative and two, for a map between surfaces with nonpositive target curvature.
\end{abstract}

\section{Introduction}

The theory of harmonic maps into smooth Riemannian manifolds of nonpositive curvature began with the work of J. Eells and H. Sampson [ES] and S.I. Al'ber [Al]. Using a heat flow approach, they constructed a harmonic map in a given homotopy class. Later, R. Hamilton was able to extend their theory to manifolds with boundary $[\mathbf{H m}]$. The uniqueness theorem for harmonic maps between Riemannian manifolds is due to Al'ber $[\mathbf{A l}]$ and P. Hartman $[\mathbf{H r}]$ :

TheOREM 1 (Uniqueness of harmonic maps). Let $M$ and $N$ be smooth Riemannian manifolds and $u_{0}, u_{1}: M \rightarrow N$ be homotopic harmonic maps which agree on $\partial M$. If $\partial M \neq \emptyset$ and $N$ has nonpositive curvature, then $u_{0} \equiv u_{1}$. If $\partial M=\emptyset$ and $N$ has negative sectional curvature, then $u_{0} \equiv u_{1}$ unless $u_{0}(M)$ is contained in a geodesic.

More recently, progress has been made on the harmonic map theory with nonsmooth target spaces. In the paper [GS], M. Gromov and R. Schoen initiated the study of harmonic maps into singular spaces by considering locally compact polyhedral targets of nonpositive curvature. This work was substantially generalized by N. Korevaar and R. Schoen $[\mathbf{K S 1}][\mathbf{K S 2}][\mathbf{K S 3}]$ and J. Jost [Jo1] [Jo2] [Jo3] [Jo4] [Jo5]. In particular, Korevaar and Schoen developed the Sobolev space theory for maps into complete metric spaces and constructed Lipschitz energy minimizing maps for the Dirichlet, homotopy, and equivariant problems. It is in this general framework that we consider the uniqueness question (see Theorem 2 and Theorem 3 below). We note that it is straightforward to extend the arguments to energy

${ }^{*}$ The author is supported by research grant NSF DMS 0072483 and by the Woodrow Wilson National Fellowship Foundation. 
minimizing maps from domains with locally bounded measurable metrics following the work of G. Gregori $[\mathbf{G}]$ or to consider maps between Riemannian polyhedra as in the work of J. Eells and B. Fuglede $[\mathbf{E F}]$. The work of Jost considers the possibility that the domain space may be a metric space equipped with a measure. Jost also claims some uniqueness results in [Jo5], but we will give a counter example to his argument (see Remark 16, Example 17, and Remark 19 below).

The key to the uniqueness theorem is the convexity of energy statement. First, consider the case where $M$ and $N$ are smooth Riemannian manifolds. Let $F$ : $M \times[0,1] \rightarrow N$ be a $C^{2}$ map and set $u_{t}(x)=F(x, t)$ for $x \in M$. We then have the following formula which can be found in $[\mathbf{E S}]$ :

$$
\frac{d^{2}}{d t^{2}} E^{u_{t}}=2 \int_{M}\left(\sum_{\alpha}\left|\nabla_{e_{\alpha}}^{\prime} V\right|^{2}-K_{N}\left(V, F_{*} e_{\alpha}\right)+<\nabla_{e_{\alpha}}^{\prime} \nabla_{\frac{\partial}{\partial t}}^{\prime} V, F_{*} e_{\alpha}>\right) d \mu .
$$

Here, $E^{u_{t}}$ is the energy of $u_{t}, V=F_{*} \frac{\partial}{\partial t}, \nabla^{\prime}$ is the pullback connection from $T N$, $K_{N}\left(V, F_{*} e_{\alpha}\right)$ is the sectional curvature of the tangent plane defined by $V$ and $F_{*} e_{\alpha}$ in $N$, and $\left\{e_{\alpha}\right\}$ is an orthonormal basis for $T M$. If $F$ is a geodesic homotopy, i.e. $t \mapsto u_{t}(x)$ is a geodesic curve, then $\nabla_{\frac{\partial}{\partial t}}^{\prime} V=0$ and the last term on the right drops off. Thus, if $N$ has nonpositive sectional curvature, the map $t \mapsto E^{u_{t}}$ is a convex function. Moreover, if $u_{0}$ and $u_{1}$ are energy minimizing in a class of homotopic maps, then $t \mapsto E^{u_{t}}$ has slope zero at 0 and 1 and hence $\frac{d^{2}}{d t^{2}} E^{u_{t}} \equiv 0$. Therefore, equation (1) implies

$$
\left|\nabla_{e_{\alpha}}^{\prime} V\right| \equiv 0 \text { and } K_{N}\left(V, F_{*} e_{\alpha}\right) \equiv 0
$$

Theorem 5 can now be proved by the following argument: The first identity implies that $|V(x)|$ is a constant. If $\partial M \neq \emptyset$, then $|V(x)|=0$ for $x \in \partial M$ since $u_{0}=u_{1}$ on $\partial M$. Thus $|V(x)|=0$ for all $x \in M$ and this implies $u_{0} \equiv u_{1}$. If $\partial M=\emptyset$ and $u_{0}, u_{1}$ are distinct maps, then $|V(x)|$ is nowhere zero. Therefore, if $N$ has negative curvature, $V$ and $F_{*} e_{\alpha}$ are parallel for all $\alpha$. Consequently, we can deduce that $u_{0}$ maps onto a geodesic.

Now let $(X, d)$ be a metric space of nonpositive curvature and consider maps $u$ : $M \rightarrow X$. The difficulty in working in the generality of metric spaces is contending with the lack of smoothness. For example, the formula given in equation (1) utilizes the curvature tensor; to define the curvature tensor in a Riemannian manifold, the Riemannian metric must be at least twice differentiable. For maps with a metric space target, we must find an alternative way to express the geometric information contained in equation (1). This obstacle can be overcome by a careful use of the quadrilateral comparison property of nonpositively curved metric spaces. The quadrilateral comparison states that for any quadrilateral $\mathcal{Q}$ in $X$ (i.e. ordered points $P, Q, R, S \in X$ and geodesics between two consecutive points), there exists a convex quadrilateral $V$ in $\mathbf{R}^{2}$ and a distance decreasing map $\varphi: V \rightarrow X$ so that $\varphi$ is an acrlength preserving map of $\partial V$ to $\mathcal{Q}$ (see $[\mathbf{R e}]$ ). By applying this property for quadrilaterals formed by $u_{0}(x), u_{1}(x), u_{1}(y), u_{0}(y) \in X, x, y \in M$, we obtain the inequality

$$
\begin{aligned}
d^{2}\left(u_{t}(x), u_{t}(y)\right) \leq(1-t) d\left(u_{0}(x), u_{0}(y)\right)+t d\left(u_{1}(x), u_{1}(x)\right) \\
-t(1-t)\left(d\left(u_{0}(x), u_{1}(x)\right)-d\left(u_{0}(y), u_{1}(y)\right)^{2} .\right.
\end{aligned}
$$


Using the Korevaar-Schoen definition of energy, this inequality leads to the energy convexity statement,

$$
E^{u_{t}} \leq(1-t) E^{u_{0}}+t E^{u_{1}}-t(1-t) \int_{M}\left|\nabla d\left(u_{0}, u_{1}\right)\right|^{2} d \mu .
$$

In [KS1], existence and regularity theorems of energy minimizing map into nonpositively curved metric spaces are proved by exploiting this type of (similar, but more general) convexity statements. The boundary regularity of these maps were proved by T. Serbinowski [Se1]. The existence and regularity of the Dirichlet problem when the target metric space has curvature bounded from above by $\kappa>0$ (with a smallness of image assumption) was also proved by T. Serbinowski [Se2]. The uniqueness of these solutions when $\partial M \neq \emptyset$ also follows from the convexity of energy statement in the analogous fashion as described above.

In this paper, we consider the uniqueness question in the case when $\partial M=\emptyset$. We will first assume that the target space has an upper curvature bound of $\kappa<0$. In the smooth case, we can deduce that the energy function is strictly convex from equation (1) if the map is not everywhere rank $\leq 1$. We will make sense of this statement in the singular setting; we improve the convexity statement of KorevaarSchoen by using the stricter curvature bound and prove the uniqueness statement without using the boundary information. In doing so, we will analyze quadrilaterals in hyperbolic surfaces and use the suitable quadrilateral comparison property. We show:

TheOREM 2. Let $M$ be a compact Riemannian manifold, $(X, d)$ be a metric space of curvature bounded from above by $\kappa<0$ and $\rho$ be an isometric action of the fundamental group $\Gamma$ of $M$ on $X$. If $u_{0}, u_{1}: \tilde{M} \rightarrow X$ are a $\rho$-equivariant energy minimizing maps, then $u_{0} \equiv u_{1}$ unless $u_{0}(M)$ is contained in a geodesic.

A special case of Theorem 2 is the following.

THEOREM 3. Let $M$ be a compact Riemannian manifold and $N$ be a compact metric space whose univeral cover $X$ is a metric space of curvature bounded from above by $\kappa<0$. If $u_{0}, u_{1}: M \rightarrow N$ are energy minimizing maps in its homotopy class, then $u_{0} \equiv u_{1}$ unless $u_{0}(M)$ is contained in a geodesic.

In the second part of the paper, we will show the uniqueness property of maps between compact surfaces. Recall a theorem of J. Jost and R. Schoen $[\mathbf{J S}]$ which states that any diffeomorphism between two Riemannian surfaces $\Sigma_{1}$ and $\Sigma_{2}$ is homotopic to a harmonic diffeomorphism. For genus $\geq 2$, J.M. Coron and F. Hélein $[\mathbf{C H}]$ showed that the Jost-Schoen diffeomorphism is the unique minimizer in its homotopy class. We will prove that this uniqueness statement is true when the target surface is equipped with a singular metric of nonpositive curvature.

THEOREM 4. Let $\left(\Sigma_{1}, z\right)$ and $\left(\Sigma_{2}, w\right)$ be compact Riemann surfaces with genus $g \geq 2$ and $\lambda|d w|^{2}$ be a possibly degenerate and non-smooth metric with $\lambda$ satisfying $\triangle \log \lambda \geq 0$ weakly. Let $\varphi: \Sigma_{1} \rightarrow \Sigma_{2}$ be a diffeomorphism. If $u_{0}, u_{1}: \Sigma_{1} \rightarrow \Sigma_{2}$ are energy minimizings homotopic to $\varphi$, then $u_{0} \equiv u_{1}$.

Metrics of the type described in Theorem 4 include flat metrics with conical singularities and cone angles $\geq 2 \pi$. For example, if $\Phi=\phi d z^{2}$ is a quadratic holomorphic differential on a Riemann surface $\Sigma_{2}$, then the metric defined by $|\phi||d z|^{2}$ is such a metric. 
In $[\mathbf{M e}]$, we show that we can construct an energy minimizing map $u$ in a homotopy class of $\varphi$ as a limit of smooth diffeomorphism. Theorem 4 shows that this is the only harmonic map in its homotopy class. The key to the proof of Theorem 4 is the analysis of the local behavior of the map $u$. Using this analysis, we show that if $u_{0}$ and $u_{1}$ are distinct energy minimizing maps, we can construct a self-map of a Riemann surface of genus $\geq 2$ homotopic to the identity without any fixed points, a contradiction.

We finish with some immediate applications of the uniqueness theorem. First is the generalization of the Preissman's Theorem. This states that every ablelian subgroup of the fundamental group of a compact metric space of curvature bounded from above by $\kappa<0$ is abelian. Here, we use the uniqueness statement in place of the Bochner's formula used in the smooth setting. Secondly, we will give a necessary condition for the existence of equivariant maps representing isometric actions of discrete groups on a negatively curved metric space. This complements the sufficient condition established independently by Korevaar and Schoen [KS3] and by Jost $[\mathbf{J o 2}]$ and generalizes the work of K. Corlette $[\mathbf{C}]$ and F. Labourie $[\mathbf{L}]$; more precisely,

THEOREM 5. If $X$ is a metric space of curvature bounded from above by $\kappa<0$, $\rho$ is an isometric action of the fundamental group $\Gamma$ of a compact Riemannian manifold $M$ on $X$, if there exists a $\rho$-equivariant harmonic map whose image is not contained in a geodesic, then there are no geodesic ray whose equivalence class is fixed by $\rho(\Gamma)$.

More generally, we can show

THEOREM 6. Let $\rho$ be an isometric action of the fundamental group $\Gamma$ of a compact Riemannian manifold $M$ on $X$, a complete metric space of nonpositive curvature, of finite rank and with no flat half-strip. There exists a $\rho$-equivariant harmonic map if and only if $\rho(\Gamma)$ is a reductive subgroup of $\operatorname{Isom}(X)$.

Here, we use the definition of no flat half-strip which adapts the definition used in $[\mathbf{L}]$ to our setting (see Definition 26).

\section{Energy of maps into metric spaces}

We first outline the Sobolev space theory for maps into metric spaces developed by Korevaar and Schoen $[\mathbf{K S 1}]$. Let $(M, g)$ be a compact Riemannian manifold, $d_{M}$ be the distance function on $M$ induced by $g$ and $(X, d)$ be a complete metric space. A Borel measurable map $f: M \rightarrow X$ is said to be in $L^{2}(M, X)$ if

$$
\int_{M} d^{2}(f(x), P) d \mu<\infty
$$

for some $P \in X$. By the triangle inequality, this definition is independent of $P$ chosen.

Let $M_{\epsilon}=\left\{x \in M: d_{M}(x, \partial M)>\epsilon\right\}, S_{\epsilon}(x)=\left\{y \in M: d_{M}(x, y)=\epsilon\right\}$ and $\omega_{n}$ be the area form of the unit sphere. For $u \in L^{2}(\Omega, X)$, construct an $\epsilon$-approximate energy function $e_{\epsilon}: M \rightarrow \mathbf{R}$ by setting

$$
e_{\epsilon}(x)= \begin{cases}\frac{1}{\omega_{n}} \int_{S_{\epsilon}(x)} \frac{d^{2}(u(x), u(y))}{\epsilon^{2}} \frac{d \sigma}{\epsilon^{n-1}} & \text { for } x \in M_{\epsilon} \\ 0 & \text { for } x \in M-M_{\epsilon} .\end{cases}
$$


Define a linear functional $E_{\epsilon}: C_{c}(M) \rightarrow \mathbf{R}$ on the set of continuous functions with compact support in $M$ by setting

$$
E_{\epsilon}(\varphi)=\int \varphi e_{\epsilon} d \mu
$$

The map $u \in L^{2}(M, X)$ has finite energy (or $\left.u \in W^{1,2}(M, X)\right)$ if

$$
E^{u} \equiv \sup _{\varphi \in C_{c}(M), 0 \leq \varphi \leq 1} \limsup _{\epsilon \rightarrow 0} E_{\epsilon}(\varphi)<\infty .
$$

The quantity $E^{u}$ is defined to be the energy of the map $u$. It can be shown that if $u$ has finite energy, the measures $e_{\epsilon}(x) d \mu(x)$ converge weakly to a measure which is absolutely continuous with respect to the Lebesgue measure. Therefore, there exists a function $e(x)$, which we call the energy density function, so that $e_{\epsilon}(x) d \mu \rightarrow e(x) d \mu$. In analogy with the case of real-valued functions and maps into Riemannian manifolds, we write $|\nabla u|^{2}(x)$ in place of $e(x)$. In particular,

$$
E^{u}=\int_{M}|\nabla u|^{2} d \mu
$$

Let $\Gamma(T M)$ be the set of Lipschitz vector field on $M$. Then for $V \in \Gamma(T M)$, the directional energy measures can be defined as the weak* limit of measures ${ }^{V} e_{\epsilon}(x) d x$ where

$$
V e_{\epsilon}(x)= \begin{cases}\frac{d^{2}(u(x), u(\bar{x}(x, \epsilon))}{\epsilon^{2}} & \text { for } x \in M_{\epsilon} \\ 0 & \text { for } x \in M-M_{\epsilon} .\end{cases}
$$

and $\bar{x}(x, \epsilon)$ denotes the flow along $V$ at time $\epsilon$ starting at point $x$. The energy density measures can be written $\left|u_{*}(V)\right|^{2} d \mu$ for an $L^{2}$ function $\left|u_{*}(V)\right|$ and for almost every $x \in M$,

$$
\lim _{\epsilon \rightarrow 0} V e_{\epsilon}(x)=\left|u_{*}(Z)\right|^{2}(x) .
$$

If $\left\{e_{1}, e_{2}, \ldots, e_{n}\right\}$ is a local orthonormal frame on $M$ and if we identify $S^{n-1} \subset \mathbf{R}^{n}$ with $S_{x}^{n-1} \subset T M_{x}$ by

$$
\omega=\left(\omega^{1}, \omega^{2}, \ldots, \omega^{n}\right) \mapsto w^{i} e_{i}
$$

then

$$
|\nabla u|^{2}(x)=\frac{1}{\omega_{n}} \int_{\omega \in S^{n-1}}\left|u_{*}(\omega)\right|^{2} d \sigma(\omega) .
$$

\section{Curvature bounds of metric spaces}

A complete metric space $(X, d)$ is said to have curvature bounded from above by $\kappa$ if its universal cover $\tilde{X}$ has the following properties:

(i) The space $(\tilde{X}, d)$ is a length space. That is, for any two points $P$ and $Q$ in $\tilde{X}$, there exists a rectifiable curve $\gamma_{P Q}$ so that the length of $\gamma_{P Q}$ is equal to $d(P, Q)$ (which we will sometimes denote by $d_{P Q}$ for simplicity). We call such distance realizing curves geodesics.

(ii) Given $P, Q, R \in \tilde{X}$ (assume $d_{P Q}+d_{Q R}+d_{R P}<\frac{\pi}{\sqrt{\kappa}}$ ) for $\kappa>0$ and geodesic $\gamma_{Q R}$ between $Q$ and $R$, let $Q_{t}$ be point of $\gamma_{Q R}$ so that $d_{Q Q_{t}}=t d_{Q R}$. Then, setting $a=\sqrt{|\kappa|}$,

$$
\cosh \left(a d_{P Q_{t}}\right) \leq \frac{\sinh \left((1-t) a d_{Q R}\right)}{\sinh \left(a d_{Q R}\right)} \cosh a d_{P Q}+\frac{\sinh \left(\operatorname{tad}_{Q R}\right)}{\sinh \left(a d_{Q R}\right)} \cosh a d_{P R}
$$


for $\kappa<0$,

for $\kappa=0$, and

$$
d_{P Q_{t}}^{2} \leq(1-t) d_{P Q}^{2}+t d_{P R}^{2}-t(1-t) d_{Q R}^{2}
$$

$$
\cos \left(a d_{P Q_{t}}\right) \geq \frac{\sin \left((1-t) a d_{Q R}\right)}{\sin \left(a d_{Q R}\right)} \cos a d_{P Q}+\frac{\sin \left(\operatorname{tad}_{Q R}\right)}{\sin \left(a d_{Q R}\right)} \cos a d_{P R}
$$

for $\kappa>0$.

Note that for $a=1$ and $t=\frac{1}{2}$ in inequality (3), we get

$$
\cosh d_{P Q_{t}} \leq \frac{\cosh d_{P Q}+\cosh d_{P R}}{2 \cosh \frac{d_{Q R}}{2}} .
$$

We will say that $X$ is NPC (nonpositively curved) if it has curvature bounded from above by 0 . A simply connected space of curvature bounded from above by $\kappa<0$ is also referred to as $C A T(\kappa)$ spaces. The following important result is due to Y.G. Reshetnyak:

THEOREM 7 (Non-expanding maps into metric spaces [Re]). Let $S_{\kappa}$ be a simply connected surface of constant curvature $\kappa$. Let $(X, d)$ be a metric space of curvature bounded from above by $\kappa$ and $\Gamma$ be a closed rectifiable curve in $X$. Then there exists a convex domain $V$ in $S_{\kappa}$ and a map $\varphi: V \rightarrow X$ such that $\varphi$ restricted to $\partial V$ is an arclength preserving parameterization of $\Gamma$ and $\varphi$ is a distance decreasing map in $V$ (i.e. $d_{S_{\kappa}}(x, y) \geq d(\varphi(x), \varphi(y))$ for $\left.x, y, \in V\right)$.

Definition 8. Let $P, Q, R, S \in X$ (with $d_{P Q}+d_{Q R}+d_{R S}+d_{S P}<\frac{\pi}{\sqrt{\kappa}}$ if $\left.\kappa>0\right)$. We will denote the union of geodesics $\gamma_{P Q}, \gamma_{Q R}, \gamma_{R S}$ and $\gamma_{S P}$ by $\mathcal{Q}(P, Q, R, S)$. Let $\varphi: V \rightarrow X$ be as in Theorem 7 with $\Gamma=\mathcal{Q}(P, Q, R, S)$ and $\tilde{P}, \tilde{Q}, \tilde{R}, \tilde{S} \in \partial V$ be points corresponding to $P, Q, R, S$ via $\varphi$. By the property of the map $\varphi$, it is clear that $\partial V$ is a union of geodesics $\gamma_{\tilde{P} \tilde{Q}}, \gamma_{\tilde{Q} \tilde{R}}, \gamma_{\tilde{R} \tilde{S}}$ and $\gamma_{\tilde{S} \tilde{P}}$. We will call the ordered set $\{\tilde{P}, \tilde{Q}, \tilde{R}, \tilde{S}\}$ a $\kappa$-subembedding of $\{P, Q, R, S\}$. It is clear that $V$ is unique up to an isometry of $S_{\kappa}$. We define $\angle_{\kappa}(Q P S)$ to be the angle made by $\gamma_{\tilde{P} \tilde{Q}}$ and $\gamma_{\tilde{P} \tilde{S}}$ in $S_{\kappa}$ and $A_{\kappa}(P, Q, R, S)$ to be the area of $V$ in $S_{\kappa}$.

The following two lemmas will be important later on.

LEMMA 9. Let $X$ be a metric space of curvature bounded from above by $\kappa \leq 0$. Let $\{P, Q, R, S\} \subset X$. For sufficiently small $D>0$, there exists $C>0$ so that if $d_{P Q}+d_{Q R}+d_{R S}+d_{S P}<D$, then

$$
A_{\kappa}(P, Q, R, S) \leq C\left(d_{P Q} d_{P S}+d_{S R} d_{S P}\right)\left(\sin \iota_{\kappa}(Q P S)+\sin \iota_{\kappa}(R S P)\right) .
$$

Proof. Let $\{\tilde{P}, \tilde{Q}, \tilde{R}, \tilde{S}\} \subset S_{\kappa}$ be a $\kappa$-subembedding and $\{\bar{P}, \bar{Q}, \bar{R}, \bar{S}\} \subset \mathbf{R}^{2}$ be a 0 -subembedding of $\{P, Q, R, S\}$. Clearly $A_{\kappa} \leq A_{0}$. By elementary geometry on the plane,

$$
A_{0}(P, Q, R, S) \leq\left(d_{P Q} d_{P S}+d_{R S} d_{P S}\right)\left(\sin \angle_{0}(Q P S)+\sin \angle_{0}(R S P)\right) .
$$

Now since $d_{P Q}+d_{Q R}+d_{R S}+d_{S P}<D$ (which implies that $\mathcal{Q}(\tilde{P}, \tilde{Q}, \tilde{R}, \tilde{S})$ is contained in a small region of $\left.S_{\kappa}\right)$, there exists $C=C(D)$ so that $\sin \angle_{0}(Q P S) \leq$ $C \sin \angle_{\kappa}(Q P S)$ and $\sin \angle_{0}(R S P) \leq C \sin \angle_{\kappa}(R S P)$. Thus,

$$
\begin{aligned}
A_{\kappa}(P, Q, R, S) & \leq A_{0}(P, Q, R, S) \\
& \leq\left(d_{P Q} d_{P S}+d_{R S} d_{P S}\right)\left(\sin \angle_{0}(Q P S)+\sin \angle_{0}(R S P)\right)
\end{aligned}
$$




$$
\leq C\left(d_{P Q} d_{P S}+d_{S R} d_{S P}\right)\left(\sin { }_{\kappa}(Q P S)+\sin \angle_{\kappa}(R S P)\right)
$$

Lemma 10. Let $X$ be a NPC (nonpositively curved) space. For $\{P, Q, R, S\} \subset$ $X$, let $P_{t}$ be the point on geodesic $\gamma_{P S}$ so that $d_{P P_{t}}=t d_{P S}$ and $Q_{t}$ be the point on $\gamma_{Q R}$ so that $d_{Q Q_{s}}=t d_{Q R}$. Then

$$
d_{P_{t} Q_{t}}^{2} \leq(1-t) d_{P Q}^{2}+t d_{R S}^{2}-t(1-t)\left(\alpha\left(d_{S P}-d_{Q R}\right)^{2}+(1-\alpha)\left(d_{R S}-d_{P Q}\right)^{2}\right) .
$$

Proof. The inequality is true if $\{P, Q, R, S\} \subset \mathbf{R}^{2}$ by an elementary computation on the plane. Hence the lemma follows by applying Theorem 7. See Corollary 2.1.3 of [KS1] for more details.

\section{Convexity of energy and energy minimizing maps}

Denote the fundamental group $\pi_{1}(M)$ by $\Gamma$ and the universal cover of $M$ by $\tilde{M}$. If $X$ is a metric space and $\rho: \Gamma \rightarrow \operatorname{isom}(X)$ a homomorphism, $\rho$ is called an isometric action of $\Gamma$ on $X$ (or a representation of $\Gamma$ ). For $\gamma \in \Gamma$, we will write $\rho(\gamma) P$ for $\rho(\gamma)(P)$. A map $u: \tilde{M} \rightarrow X$ is said to be $\Gamma$-equivariant if

$$
u(\gamma x)=\rho(\gamma) u(x)
$$

for all $x \in \tilde{M}$ and $\gamma \in \Gamma$. The energy of the map $u$ is defined to be

$$
E^{u}=\int_{M}|\nabla u|^{2} d \mu
$$

This integral is well-defined as long as $M$ has finite volume. The map $u$ is energy minimizing if $E^{u} \leq E^{v}$ for any $\Gamma$-equivariant map $v: \tilde{M} \rightarrow X$. Commonly, a map is called harmonic if it is stationary for the energy functional. Because of the convexity of energy for maps with NPC targets (Theorem 11 below), stationary implies minimizing. Hence, we will refer to energy minimizing maps as harmonic maps. Assume $X$ is simply connected and suppose $u_{0}, u_{1}: \tilde{M} \rightarrow X$ are $\Gamma$-equivariant finite energy maps. Let $t \mapsto u_{t}(x), 0 \leq t \leq 1$, be the constant speed parameterization of the geodesic from $u_{0}(x)$ to $u_{1}(x)$. Then $u_{t}: \tilde{M} \rightarrow X$ is $\Gamma$-equivariant and the function $d\left(u_{0}, u_{1}\right)(x)=d\left(u_{0}(x), u_{1}(x)\right)$ is $\Gamma$-invariant. The following theorem is a consequence of inequality (5). We refer to $[\mathbf{K S 1}]$ for the proof.

Theorem 11 (Convexity of energy [KS1]). Let $u_{0}, u_{1}$ be finite energy $\Gamma$ equivariant maps and $u_{t}$ as above. Then

$$
E^{u_{t}} \leq(1-t) E^{u_{0}}+t E^{u_{1}}-t(1-t) \int_{M}\left|\nabla d\left(u_{0}, u_{1}\right)\right|^{2} d \mu .
$$

The following corollaries follow immediately from Theorem 11.

COROllary 12. If $u_{0}$ and $u_{1}$ are harmonic maps, then $d\left(u_{0}, u_{1}\right)$ is constant for all $x \in \tilde{M}$ and $E^{u_{t}} \equiv E^{u_{0}}$ for all $0 \leq t \leq 1$.

Proof. Since $u_{0}$ and $u_{1}$ are energy minimizing,

$$
(1-t) E^{u_{0}}+t E^{u_{1}} \leq E^{u_{t}} \leq(1-t) E^{u_{0}}+t E^{u_{1}}-t(1-t) \int_{M}\left|\nabla d\left(u_{0}, u_{1}\right)\right|^{2} d \mu .
$$

Thus,

$$
\int_{M}\left|\nabla d\left(u_{0}, u_{1}\right)\right|^{2} d \mu=0
$$


and this implies $\nabla d\left(u_{0}, u_{1}\right)=0$ a.e. $x \in \tilde{M}$. Hence, $d\left(u_{0}, u_{1}\right)$ is constant. Furthermore, we see that $E^{u_{t}}=(1-t) E^{u_{0}}+t E^{u_{1}}=E^{u_{0}}$ and this shows $E^{u_{t}}$ is constant for all $t$.

Corollary 13. If $u_{0}$ and $u_{1}$ are enery minimizing maps, then $\left|\left(u_{t}\right)_{*}(V)\right|=$ $\left|\left(u_{0}\right)_{*}(V)\right|$ for any $t \in[0,1]$ and $V \in \Gamma(T \tilde{M})$.

Proof. Let $P=u_{0}(x), Q=u_{1}(x), R=u_{1}(\bar{x}(x, \epsilon)), S=u_{0}(\bar{x}(x, \epsilon))$ in inequality (5) with $\alpha=0$. If $d_{t}(x)=d\left(u_{t}(x), u_{t}(\bar{x}(x, \epsilon))\right.$, then

$$
d_{t}^{2}(x) \leq(1-t) d_{0}^{2}(x)+t d_{1}^{2}(x)-t(1-t)\left(d_{0}(x)-d_{1}(x)\right)^{2} .
$$

Dividing by $\epsilon^{2}$ and letting $\epsilon \rightarrow 0$, this implies

$\left|\left(u_{t}\right)_{*}(V)\right|^{2} \leq(1-t)\left|\left(u_{0}\right)_{*}(V)\right|^{2}+t\left|\left(u_{1}\right)_{*}(V)\right|^{2}-t(t-1)\left(\left|\left(u_{1}\right)_{*}\right|(V)-\left|\left(u_{0}\right)_{*}(V)\right|\right)^{2}$.

Since $E^{u_{t}}=E^{u_{0}}$, we must have $\left|\left(u_{t}\right)_{*}(V)\right|^{2}=(1-t)\left|\left(u_{0}\right)_{*}(V)\right|^{2}+t\left|\left(u_{1}\right)_{*}(V)\right|^{2}$. Thus, $\left|\left(u_{1}\right)_{*}\right|(V)=\left|\left(u_{0}\right)_{*}(V)\right|$.

\section{Uniqueness property when the target has curvature bounded from above by $\kappa<0$.}

If $(X, d)$ has curvature bounded from above by $\kappa<0$, then the metric space $(X, \sqrt{-\kappa} d)$ has curvature bounded from above by -1 . Hence, it is sufficient to assume that our metric space has curvature bounded from above by -1 . Metric spaces of negative curvature can be compactified by equivalence classes of geodesic rays (where a geodesic ray is an image of a map $\sigma:[0, \infty) \rightarrow X$ with the property that $\left.d\left(\sigma\left(t_{1}\right), \sigma\left(t_{2}\right)\right)=\left|t_{1}-t_{2}\right|\right)$. Two geodesic rays $\sigma_{1}, \sigma_{2}$ are said to be equivalent if the Hausdorff distance between them is finite. We have the following dichotomy for the existence question of harmonic equivariant maps:

TheOREM 14 (Existence of a harmonic equivariant map [KS2]). Let $M$ be a compact Riemannian manifold without boundary, $X$ be a metric space of curvature bounded from above by -1 and $\rho: \Gamma \rightarrow$ isom $(X)$ be an isometric action of the fundamental group $\Gamma$ of $M$ on $X$. Either there is a geodesic ray in $X$ whose equivalence class is fixed by $\Gamma$, or there is a harmonic equivariant map from $\tilde{M}$ to $X$.

Our goal in this section is to prove Theorem 2, the uniqueness of equivariant harmonic maps when it exists. We consider a model of $S_{-1}$, the hyperbolic plane, by taking the dot product $\cdot$ on $\mathbf{R}^{3}$ defined by $\left(x_{0}, y_{0}, z_{0}\right) \cdot\left(x_{1}, y_{1}, z_{1}\right)=-x_{0} x_{1}+$ $y_{0} y_{1}+z_{0} z_{1}$ and a subset $\mathbf{H}^{2}=\left\{(x, y, z) \subset \mathbf{R}^{3}:-x^{2}+y^{2}+z^{2}=-1, x>0\right\}$. Let $P, Q, R, S$ be a points in $\mathbf{H}^{2}$ and let $\alpha=\angle Q P S$ and $\beta=\angle R S P$. Without the loss of generality, we assume $P=(1,0,0)$ and $S=(\cosh \theta, \sinh \theta, 0)$. (Note that $\cosh d_{\mathbf{H}^{2}}(P, S)=-Q \cdot R=\cosh \theta$ and hence $d_{\mathbf{H}^{2}}(P, S)=\theta$.) Let $\varphi(t)=$ $(\cosh t, \sinh t, 0)$; that is, $\varphi$ is the unit speed geodesic from $P$ to $S$. Furthermore, let $\gamma($ resp. $\sigma$ ) be the unit speed geodesic from $P$ (resp. $S$ ) to $Q$ (resp. $R$ ). We can check that

$$
\gamma(t)=(\cosh t, \cos \alpha \sinh t, \sin \alpha \sinh t)
$$

and

$\sigma(s)=(-\cos \beta \sinh \theta \sinh s+\cosh \theta \cosh s,-\cos \beta \cosh \theta \sinh s+\sinh \theta \cosh s, \sin \beta \sinh s)$. 
We now compute $\Theta=d_{\mathbf{H}^{2}}(\gamma(t), \sigma(s))$ in terms of $t, s, \alpha, \beta$ and $\theta$.

$$
\begin{aligned}
-\cosh \Theta= & \gamma(t) \cdot \sigma(s) \\
= & \cosh t \sinh s \sinh \theta \cos \beta-\cosh t \cosh s \cosh \theta \\
& -\sinh t \sinh s \cosh \theta \cos \alpha \cos \beta+\cosh s \sinh t \sinh \theta \cos \alpha \\
& +\sinh t \sinh s \sin \alpha \sin \beta .
\end{aligned}
$$

Rearranging terms, we obtain

$$
\begin{aligned}
- & \cosh s \sinh t \sinh \theta \cos \alpha-\cosh t \sinh s \sinh \theta \cos \beta \\
= & \cosh \Theta-\cosh t \cosh s \cosh \theta \\
& -\sinh t \sinh s \cosh \theta \cos \alpha \cos \beta+\sinh t \sinh s \sin \alpha \sin \beta .
\end{aligned}
$$

We now expand some of the terms of the equation in variables $t$ and $s$ and denote terms which are of order higher than degree 2 in $t$ and $s$ by $o^{2}(t, s)$.

$$
\begin{aligned}
- & \sinh t \sinh \theta \cos \alpha-\sinh s \sinh \theta \cos \beta \\
= & \cosh \Theta-\cosh \theta\left(1+\frac{1}{2}\left(t^{2}+s^{2}\right)\right) \\
& \quad-t s \cosh \theta \cos \alpha \cos \beta+t s \sin \alpha \sin \beta+o^{2}(t, s) .
\end{aligned}
$$

Now assume $\theta=\Theta$. (In the application below, we will use $\theta=d\left(u_{0}(x), u_{1}(x)\right)$ and $\Theta=d\left(u_{0}(\bar{x}(x, \epsilon)), u_{1}(\bar{x}(x, \epsilon))\right)$ so setting $\theta=\Theta$ is justified by Corollary 12.) Then

$$
\begin{aligned}
-\sinh & t \sinh \theta \cos \alpha-\sinh s \sinh \theta \cos \beta \\
= & -\frac{1}{2}\left(t^{2}+s^{2}\right) \cosh \theta \\
& -t s \cosh \theta \cos \alpha \cos \beta+t s \sin \alpha \cos \beta+o^{2}(t, s) \\
= & -\left(\frac{1}{2}\left(t^{2}+s^{2}\right)+t s \cos \alpha \cos \beta\right) \cosh \theta \\
& +t s \sin \alpha \sin \beta+o^{2}(t, s) .
\end{aligned}
$$

Adding and subtracting $-\left(\frac{1}{2}\left(t^{2}+s^{2}\right)+t s \cos \alpha \cos \beta\right)$ to the right side of the equation, we obtain

$$
\begin{aligned}
-\sinh & t \sinh \theta \cos \alpha-\sinh s \sinh \theta \cos \beta \\
=- & \left(\frac{1}{2}\left(t^{2}+s^{2}\right)+t s \cos \alpha \cos \beta\right)(\cosh \theta-1) \\
- & \left(\frac{1}{2}\left(t^{2}+s^{2}\right)+t s \cos \alpha \cos \beta\right)+t s \sin \alpha \sin \beta+o^{2}(t, s) .
\end{aligned}
$$

Using the trig identity $\cos \alpha \cos \beta-\sin \alpha \sin \beta=\cos (\alpha+\beta)$, we obtain

$$
\begin{aligned}
-\sinh & t \sinh \theta \cos \alpha-\sinh s \sinh \theta \cos \beta \\
= & -\left(\frac{1}{2}\left(t^{2}+s^{2}\right)+t s \cos \alpha \cos \beta\right)(\cosh \theta-1) \\
- & \frac{1}{2}\left(t^{2}+s^{2}\right)-t s \cos (\alpha+\beta)+o^{2}(t, s) .
\end{aligned}
$$


Adding and subtracting $t s(\cosh \theta-1)$ and $t s$ to the right hand side of the equation, we obtain

$$
\begin{aligned}
-\sinh & t \sinh \theta \cos \alpha-\sinh s \sinh \theta \cos \beta \\
= & -\frac{1}{2}\left(t^{2}+s^{2}-2 t s\right)(\cosh \theta-1)-t s(1+\cos \alpha \cos \beta)(\cosh \theta-1) \\
& \quad-\frac{1}{2}\left(t^{2}+s^{2}-2 t s\right)-t s(1+\cos (\alpha+\beta))+o^{2}(t, s) \\
= & -\frac{1}{2}(t-s)^{2}(\cosh \theta-1)-\frac{1}{2}(t-s)^{2} \\
& \quad-t s(1+\cos \alpha \cos \beta)(\cosh \theta-1)-t s(1+\cos (\alpha+\beta))+o^{2}(t, s) .
\end{aligned}
$$

Therefore,

$$
\begin{aligned}
& -\sinh t \sinh \theta \cos \alpha-\sinh s \sinh \theta \cos \beta \\
& =\quad-\frac{1}{2}(t-s)^{2} \cosh \theta \\
& \quad-t s((1+\cos \alpha \cos \beta)(\cosh \theta-1)-(1+\cos (\alpha+\beta)))+o^{2}(t, s) .
\end{aligned}
$$

Let $V \in \Gamma(T \tilde{M})$ and $\bar{x}(x, \epsilon)$ as before. We will denote $d\left(u_{t}(x), u_{t}(\bar{x}(x, \epsilon))\right.$ by $d_{t}(x)$ and $d\left(u_{0}, u_{1}\right)$ by $\theta$. The triangle inequality (4) applied to geodesic triangle defined by $\left\{u_{\frac{1}{2}}(x), u_{0}(\bar{x}(x, \epsilon)), u_{1}(\bar{x}(x, \epsilon))\right\}$ gives us

$$
2 \cosh d_{\frac{1}{2}} \leq \frac{1}{\cosh \frac{\theta}{2}}\left(\cosh d\left(u_{\frac{1}{2}}(x), u_{0}(\bar{x}(x, \epsilon))\right)+\cosh d\left(u_{\frac{1}{2}}(x), u_{1}(\bar{x}(x, \epsilon))\right)\right) .
$$

Now let $\{P, Q, R, S\} \subset \mathbf{H}^{2}$ be a hyperbolic subembedding of

$$
\left\{u_{0}(x), u_{0}(\bar{x}(x, \epsilon)), u_{1}(\bar{x}(x, \epsilon)), u_{1}(x)\right\} .
$$

Let $\alpha(x, \epsilon)=L_{-1}\left(u_{0}(\bar{x}(x, \epsilon)) u_{0}(x) u_{1}(x)\right)$ and $\beta(x, \epsilon)=L_{-1}\left(u_{1}(\bar{x}(x, \epsilon)) u_{1}(x) u_{0}(x)\right)$. (Recall that $L_{\kappa}(Q P S)$ is the angle at $\tilde{P}$ of the quadrilateral defined by the $\kappa$ subembedding $\{\tilde{P}, \tilde{Q}, \tilde{R}, \tilde{S}\} \subset S_{\kappa}$ of $\{P, Q, R, S\} \subset X$.) Then by the law of cosines for the hyperbolic surface applied to triangles $\triangle P Q P_{\frac{1}{2}}$ and $\triangle S R P_{\frac{1}{2}}$, we have

$$
\begin{aligned}
\cosh d\left(u_{\frac{1}{2}}(x), u_{0}(\bar{x}(x, \epsilon))\right)+\cosh d\left(u_{\frac{1}{2}}(x), u_{1}(\bar{x}(x, \epsilon))\right) \\
\leq \quad \cosh d_{0} \cosh \frac{\theta}{2}-\sinh d_{0} \sinh \frac{\theta}{2} \cos \alpha(x, \epsilon) \\
\quad+\cosh d_{1} \cosh \frac{\theta}{2}-\sinh d_{1} \sinh \frac{\theta}{2} \cos \beta(x, \epsilon) \\
=\quad\left(\cosh d_{0}+\cosh d_{1}\right) \cosh \frac{\theta}{2} \\
\quad-\frac{1}{2 \cosh \frac{\theta}{2}}\left(\sinh d_{0} \sinh \theta \cosh \alpha(x, \epsilon)+\sinh d_{1} \sinh \theta \cos \beta(x, \epsilon)\right)
\end{aligned}
$$

Applying equation (6), we obtain

$$
\begin{gathered}
\cosh d\left(u_{\frac{1}{2}}(x), u_{0}(\bar{x}(x, \epsilon))\right)+\cosh d\left(u_{\frac{1}{2}}(x), u_{1}(\bar{x}(x, \epsilon))\right) \\
\leq \quad\left(\cosh d_{0}+\cosh d_{1}\right) \cosh \frac{\theta}{2}-\frac{\cosh \theta}{4 \cosh \frac{\theta}{2}}\left(d_{0}-d_{1}\right)^{2} \\
-\frac{d_{0} d_{1}}{2 \cosh \frac{\theta}{2}} T(V, x, \epsilon)+o^{2}\left(d_{0}, d_{1}\right)
\end{gathered}
$$


where

$$
T(V, x, \epsilon)=(1+\cos \alpha(x, \epsilon) \cos \beta(x, \epsilon))(\cosh \theta-1)+(1+\cos (\alpha(x, \epsilon)+\beta(x, \epsilon))) .
$$

Together with inequality (7), this implies

$$
\begin{aligned}
2 \cosh d_{\frac{1}{2}} \leq & \cosh d_{0}+\cosh d_{1}-\frac{\cosh \theta}{4 \cosh ^{2} \frac{\theta}{2}}\left(d_{0}-d_{1}\right)^{2} \\
& -\frac{d_{0} d_{1}}{2 \cosh ^{2} \frac{\theta}{2}} T(V, x, \epsilon)+o^{2}\left(d_{0}, d_{1}\right)
\end{aligned}
$$

This shows that

$$
2 d_{\frac{1}{2}}^{2} \leq d_{0}^{2}+d_{1}^{2}-\frac{\cosh \theta}{4 \cosh ^{2} \frac{\theta}{2}}\left(d_{0}-d_{1}\right)^{2}-\frac{d_{0} d_{1}}{2 \cosh ^{2} \frac{\theta}{2}} T(V, x, \epsilon)+o^{2}\left(d_{0}, d_{1}\right)
$$

We now claim that for a.e. $x \in \tilde{M}$, either

$$
\left|\left(u_{0}\right)_{*}(V)\right|^{2}(x)=\left|\left(u_{1}\right)_{*}(V)\right|^{2}(x)=0
$$

or the following: for any sequence $\epsilon_{i} \rightarrow 0$ so that

$$
\lim _{i \rightarrow \infty} \alpha\left(x, \epsilon_{i}\right)=a \text { and } \lim _{i \rightarrow \infty} \beta\left(x, \epsilon_{i}\right)=b
$$

we either have

$$
\lim _{\epsilon \rightarrow 0} \alpha(x, \epsilon)=0 \text { and } \lim _{\epsilon \rightarrow 0} \beta(x, \epsilon)=\pi
$$

or

$$
\lim _{\epsilon \rightarrow 0} \alpha(x, \epsilon)=\pi \text { and } \lim _{\epsilon \rightarrow 0} \beta(x, \epsilon)=0 .
$$

Suppose that this claim is not true. Then there exists a set $A$ of positive measure where $\left|\left(u_{0}\right)_{*}(V)\right|^{2}=\left|\left(u_{1}\right)_{*}(V)\right|^{2} \neq 0$ and, for each $x \in A$, there exists a sequence $\epsilon_{i} \rightarrow 0$ so that $\lim _{i \rightarrow \infty} \alpha\left(x, \epsilon_{i}\right)=a$ and $\lim _{i \rightarrow \infty} \beta(x, \epsilon)=b$ with

$$
a \neq 0 \text { or } b \neq \pi
$$

and

$$
a \neq \pi \text { or } b \neq 0 \text {. }
$$

Hence,

$$
T=\lim _{\epsilon_{i} \rightarrow 0} T\left(V, x, \epsilon_{i}\right)=(1+\cos a \cos b)(\cosh \theta-1)+(1+\cos (a+b))>0 .
$$

Replacing $\epsilon$ by $\epsilon_{i}$ in inquality (8), dividing by $\epsilon_{i}^{2}$, letting $\epsilon_{i} \rightarrow 0$, and noting that

$$
\lim _{\epsilon \rightarrow 0} \frac{\left(d_{0}-d_{1}\right)^{2}}{\epsilon^{2}}=\left(\left|\left(u_{0}\right)_{*}(V)\right|-\left|\left(u_{1}\right)_{*}(V)\right|\right)^{2}=0
$$

by Corollary 13, we obtain

$$
\begin{aligned}
2\left|\left(u_{\frac{1}{2}}\right)_{*}(V)\right|^{2} \leq & \left|\left(u_{0}\right)_{*}(V)\right|^{2}+\left|\left(u_{1}\right)_{*}(V)\right|^{2} \\
& -\frac{1}{2 \cosh ^{2} \frac{\theta}{2}}\left|\left(u_{0}\right)_{*}(V)\right|\left|\left(u_{1}\right)_{*}(V)\right| T \\
< & \left|\left(u_{0}\right)_{*}(V)\right|^{2}+\left|\left(u_{1}\right)_{*}(V)\right|^{2}
\end{aligned}
$$

for a.e. $x \in A$. This contradicts Corollary 13 and we have proved our claim.

Choose an orthonormal frame in a coordinate neighborhood $U \subset \tilde{M}$. Then for a.e. $x \in U$ and a.e. $\omega \in S_{x}^{n-1}$, we have either

$$
\left|\left(u_{0}\right)_{*}(\omega)\right|=\left|\left(u_{1}\right)_{*}(\omega)\right|=0
$$


or

$$
\lim _{\epsilon \rightarrow 0} \sin \alpha(x, \epsilon)=0 \text { and } \lim _{\epsilon \rightarrow 0} \sin \beta(x, \epsilon)=0 .
$$

Let $U^{\prime} \subset U \times S^{n-1}$ be the set of pairs $(x, \omega)$ which satisfies the above.

Let $\gamma_{x y}:\left[0, t_{y}\right] \rightarrow \tilde{M}$ be an arclength parameterization of a geodesic between $x$ and $y$ (with $\left.d_{M}(x, y)=t_{y}\right)$. For a fixed $x \in U$ and for a.e. $y \in B_{r}(x)=\{z \in$ $\left.M: d_{M}(x, z)<r\right\} \subset U$, we see that $\left(\gamma_{x y}(t), \gamma_{x y}^{\prime}(t)\right) \in U^{\prime}$ for a.e. $t \in\left[0, t_{y}\right]$. Choose such $y$ and let $x_{t}=\gamma_{x y}(t)$ and $\omega_{t}=\gamma_{x y}^{\prime}(t)$.

Define $B(t, s)$ to be $A_{-1}\left(u_{0}\left(x_{t}\right), u_{1}\left(x_{t}\right), u_{1}\left(x_{s}\right), u_{0}\left(x_{s}\right)\right)$ and let $\phi(t)=B(t, 0)$. (Recall that $A_{\kappa}(P, Q, R, S)$ is the area of the quadrilateral defined by $\kappa$-subembedding $\{\tilde{P}, \tilde{Q}, \tilde{R}, \tilde{S}\} \subset S_{\kappa}$ of $\{P, Q, R, S\} \subset X$ by Definition 8.) First, we claim that $\lim _{\epsilon \rightarrow 0} \frac{B(t+\epsilon, t)}{\epsilon}=0$. To see this, we let $\alpha_{t}(\epsilon)=\alpha\left(\omega_{t}, x_{t}, \epsilon\right)$ and $\beta_{t}(\epsilon)=\beta\left(\omega_{t}, x_{t}, \epsilon\right)$. Again, we let $\theta=d\left(u_{0}, u_{1}\right)$. By Lemma 9,

$$
\begin{aligned}
& A_{-1}\left(u_{0}\left(x_{t}\right), u_{1}\left(x_{t}\right), u_{1}\left(x_{s}\right), u_{0}\left(x_{s}\right)\right) \\
& \quad \leq C\left(d\left(u_{0}\left(x_{t}\right), u_{0}\left(x_{t+\epsilon}\right)\right) \theta+d\left(u_{1}\left(x_{t}\right), u_{1}\left(x_{t+\epsilon}\right)\right) \theta\right)\left(\sin \alpha_{t}(\epsilon)+\theta \sin \beta_{t}(\epsilon)\right)
\end{aligned}
$$

Thus,

$$
\begin{aligned}
\lim _{\epsilon \rightarrow 0} & \frac{B(t+\epsilon, t)}{\epsilon} \\
\leq & \leq \lim _{\epsilon \rightarrow 0}\left(\frac{d\left(u_{0}\left(x_{t}\right), u_{0}\left(x_{t+\epsilon}\right)\right)}{\epsilon}+\frac{d\left(u_{1}\left(x_{t}\right), u_{1}\left(x_{t+\epsilon}\right)\right)}{\epsilon}\right) \theta\left(\sin \alpha_{t}(\epsilon)+\sin \beta_{t}(\epsilon)\right) \\
& \leq C\left(\left|\left(u_{0}\right)_{*}\left(\omega_{t}\right)\right|\left(x_{t}\right)+\left|\left(u_{1}\right)_{*}\left(\omega_{t}\right)\right|\left(x_{t}\right)\right) \theta \lim _{\epsilon \rightarrow 0}\left(\sin \alpha_{t}(\epsilon)+\sin \beta_{t}(\epsilon)\right) \\
& =0
\end{aligned}
$$

for a.e. $t \in\left[0, t_{y}\right]$. Now let $\phi(t)=B(t, 0)$. Then

$$
\limsup _{\epsilon \rightarrow 0} \frac{\phi(t+\epsilon)-\phi(t)}{\epsilon}=\limsup _{\epsilon \rightarrow 0} \frac{B(t+\epsilon, 0)-B(t, 0)}{\epsilon}=\limsup _{\epsilon \rightarrow 0} \frac{B(t+\epsilon, t)}{\epsilon} .
$$

Therefore, $\phi^{\prime}(t)$ exists for a.e. $t \in\left[0, t_{y}\right]$ and is equal to 0 . Thus $B\left(t_{y}, 0\right)=\phi\left(t_{y}\right)=$ $\int_{0}^{t_{y}} \phi^{\prime}(t) d t=0$ which implies that

$$
A_{-1}\left(u_{0}(y), u_{1}(y), u_{1}(x), u_{0}(x)\right)=0 .
$$

Hence, it must be that the union of geodesics $\gamma_{u_{0}(x) u_{1}(x)}$ and $\gamma_{u_{0}(y) u_{1}(y)}$ is another geodesic. By continuity, this is true for any $y \in U, u_{0}(U)$ is contained in a geodesic. This implies that $u_{0}(\tilde{M})$ is contained in the geodesic.

REMARK 15. Let $N$ be a compact metric space whose universal cover $X$ is a metric space of curvature bounded from above by $\kappa<0$. Let $M$ be a compact Riemannian manifold without boundary and $f: M \rightarrow N$ be a continuous map. For a fixed $x \in M$, the map $f$ induces the homomorphism $f_{*}: \pi_{1}(M)_{x} \rightarrow \pi(N)_{f(x)}$ and we may lift $f$ to a map $\tilde{f}: \tilde{M} \rightarrow X$ so $\tilde{f}$ is $f_{*}$ equivariant. If $u_{0}, u_{1}: M \rightarrow N$ are harmonic maps homotopic to $f$, then the lift $\tilde{u}_{0}, \tilde{u}_{1}: \tilde{M} \rightarrow X$ are harmonic $f_{*^{-}}$ equivariant maps. Hence, the uniqueness statement for harmonic maps homotopic to $f$ (Theorem 3 ) follows from the uniqueness of $f_{*}$-equivariant harmonic maps.

REMARK 16. In [Jo5], Jost also claims a uniqueness result for energy minimizing maps. But his proof is based on the assertion that if $X$ is a NPC space space and $u_{0}, u_{1}: M \rightarrow X$ are energy minimizing maps, then $d\left(u_{0}(x), u_{0}(y)\right)=d\left(u_{1}(x), u_{1}(y)\right)$ for $x, y \in M$. This assertion is incorrect as the following example shows. Note that, on the contrary, the corresponding infinitesmal statement (Corollary 13) is true. 
ExAmple 17. Let $\iota: \Sigma \rightarrow N$ be a harmonic map from a compact Riemann surface $\Sigma$ to a compact Riemannian 3-manifold $(N, g)$ of negative sectional curvature with the following properties:

(1) $\iota$ is an immersion, and

(2) $\iota(\Sigma)$ is not totally geodesic.

The existence of such a map $\iota$ is well known, see for example [SY]. By using the exponential map, we can extend $\iota$ to be an immersion $I$ from a neighborhood $O$ of the zero section in the normal bundle of $\Sigma$ where we identify the zero section with $\Sigma$. The map $I$ is a local diffeomorphism and we can pull back $g$ to $O$ using $I$. Since $\iota$ is a harmonic map, it is clear that the map $\phi: \Sigma \rightarrow\left(O, I^{*} g\right)$ defined to be just the identity map of $\Sigma$ to the copy of $\Sigma$ in $O$ is also a harmonic map.

Let $d_{g}(\cdot, \cdot)$ be the distance function on $O$ induced by the metric $I^{*} g$ and let

$$
\Sigma_{t}=\left\{x \in O: d_{g}(x, \Sigma) \leq t\right\} .
$$

Then for sufficiently small values of $t$, the boundary $\partial \Sigma_{t}$ is a smooth surface containing two components which we will denote $\partial \Sigma_{t}^{+}$and $\partial \Sigma_{t}^{-}$. Let $\Pi: O \rightarrow \Sigma$ be the canonical projection map and $\Phi_{t}: \Sigma \rightarrow O$ be the map defined by

$$
\Phi_{t}(x)=\left\{\begin{array}{cc}
\Pi^{-1}(x) \cap \partial \Sigma_{t}^{+} & \text {for } t>0 \\
x & \text { for } t=0 \\
\Pi^{-1}(x) \cap \partial \Sigma_{t}^{-} & \text {for } t<0
\end{array}\right.
$$

Since $N$, and hence $O$, has negative sectional curvature, the curvature of $\Sigma$ is negative by a theorem of Sampson [Sa]. Therefore, for sufficiently small $t$ (say for $\left.t \leq t_{0}\right), \partial \Sigma_{t}$ has nonpositive curvature.

We now note the following theorem of Alexander, Berg, and Bishop [ABB] which gives a criterion of when a Riemannian manifold with boundary is a metric space of curvature bounded from above:

Theorem 18 (Alexander, Berg, Bishop). Let $M$ be a smooth Riemannian manifold with a smooth boundary $B$ and $\rho_{0}(\cdot, \cdot)$ be the induced distance function on $M$ (i.e. for $x, y \in M, \rho_{0}(x, y)$ is the infimum of the lengths of curves in $M$ that joins these points). The sectional curvature of the interior of $M$ and the sectional curvature of the tangent sections of the boundary $B$ which bends away from the interior (i.e. 2-planes in the tangent space all of whose normal curvature vectors point outward) are bounded from above by $\kappa$ if and only if $(M, \rho)$ is a metric space of curvature bounded from above by $\kappa$.

For example, consider $\Sigma_{t} \subset O$ for $t \leq t_{0}$ with $\rho_{0}$ defined as in Theorem 18 . It follows from Theorem 18 that it is a NPC space since $\Sigma_{t}$ has negative sectional curvature in the interior and $\partial \Sigma_{t}$ is nonpositively curved. The space $Y=\Sigma_{t} \times \mathbf{R}$ with the distance function $\rho$ defined by $\rho^{2}(p, q)=\rho_{0}^{2}(x, y)+|t-s|^{2}$ for $p=(x, t), q=$ $(y, s) \in Y$ is a nonpositively curved metric space since it is a product of two nonpositively curved spaces.

We define

Let $f:[0, \infty) \rightarrow\left[0, t_{0}\right)$ be an increasing function with $f(0)=0$ and $f^{\prime \prime}(x)<0$.

$$
X=\left\{(x, t) \in \Sigma_{t_{0}} \times[0, \infty): x \in \Sigma_{f(t)}\right\} \subset Y .
$$

Let $d(\cdot, \cdot)$ be the distance funtion defined by taking the infimum of lengths with respect to $\rho$ of curves in $X$. It is easy to check that $(X, d)$ is a length space. We 
point out some properties of $(X, d)$ that will be important.

(i) For $p=(x, t)$ in the interior of $X$, there is a small neighborhood $U \subset O$ of $x$ and a sufficiently small $\epsilon>0$ so that $U \times(t-\epsilon, t+\epsilon)$ is isometrically embedded in a neighborhood of $p$. Here $U$ comes with the Riemannian metric $I^{*} g$.

(ii) For any $p, q \in X, d(p, q) \geq \rho(p, q)$. Equality holds if and only if the geodesic from $p$ to $q$ in $X$ is the same as that in $Y$.

(iii) For $p_{t}=(x, t), q_{t}=(y, t) \in \Sigma_{f(t)} \times\{t\} \subset X$ and $p_{s}=(x, s), q_{s}=(y, s) \in$ $\Sigma_{f(s)} \times\{s\} \subset X, d\left(p_{t}, q_{t}\right) \geq d\left(p_{s}, q_{s}\right)$ when $t \leq s$. We give two examples to illustrate this point. First, if the geodesics from $p_{t}$ to $q_{t}$ in $Y$ and the geodesic from $p_{s}$ to $q_{s}$ in $Y$ are the same as those in $X$, then we have $d\left(p_{t}, q_{t}\right)=\rho\left(p_{t}, q_{t}\right)=$ $\rho\left(p_{s}, q_{s}\right)=d\left(p_{s}, q_{s}\right)$. Second, if the geodesic from $p_{s}$ to $q_{s}$ in $Y$ is the same as that in $X$, but if some part of the geodesic from $p_{t}$ to $q_{t}$ in $Y$ lies outside of $X$, then $d\left(p_{t}, q_{t}\right)>\rho\left(p_{t}, q_{t}\right)=\rho\left(p_{s}, q_{s}\right)=d\left(p_{s}, q_{s}\right)$.

We now wish to apply Theorem 18 to show that $X$ is a NPC space. Unfortunately, $X$ does not satisfy the hypothesis of Theorem 18 since $\partial X$ is not smooth. In fact, $\partial X$ consists of two parts $\partial X^{1}$ and $\partial X^{2}$ where

$$
\partial X^{1}=\Sigma \times\{0\} \text { and } \partial X^{2}=\left\{(x, t): x \in \partial \Sigma_{f(t)}, t>0\right\} .
$$

Note that $\partial X^{2}$ is a smooth manifold of dimension three while $\partial X^{1}$ is two dimensional; thus $\partial X^{1}$ is the singular set of $\partial X$.

On the other hand, there is no difficulty in applying the analysis of $[\mathbf{A B B}]$ to our situation. A crucial step in their analysis is to check that all geodesics which touch the boundary and has vanishing acceleration satisfy a certain condition (that all its normal generalized Jacobi fields are 0-convex, see $[\mathbf{A B B}]$ for precise definitions); geodesics that lie in the interior of $X$ automatically satisfyies this condition. In our case, we show, by proving the following claim, that we need not be concerned with $\partial X^{1}$ :

Claim: For any pair of points $p, q \in X-\partial X^{1}$, the geodesic $\gamma$ between them does not touch $\partial X^{1}$.

Proof. Let $p=(x, t), q=(y, s) \in X-\partial X^{1}$ and assume $t \leq s$. Define a map $\Psi: X \rightarrow X$ to be the projection map of $X$ onto $X(t)=\{(z, \tau) \in X: \tau \geq t\}$, i.e.

$$
\Psi(z, \tau)= \begin{cases}(z, t) & \text { for } \tau<t \\ (z, \tau) & \text { for } \tau \geq t\end{cases}
$$

Let $\gamma:[0,1] \rightarrow X$ be a geodesic between $p$ and $q$. Then by property (iii), the curve $\Psi \circ \gamma$ has length less than $\gamma$ unless $\gamma([0,1]) \subset X(t)$. This shows $\gamma$ does not intersect $\partial X^{1}$.

The claim shows that $\partial X^{1}$ does not play a role when applying the analysis of $[\mathbf{A B B}]$ and we are left to check that the sectional curvatures of the tangent 2 -setions of $\partial X^{2}$ which bends away from the interior has curvature $\leq 0$.

Fix $\left(x_{0}, t_{0}\right) \in \partial X^{2}$. There are two types of 2-planes in $T_{\left(x_{0}, t_{0}\right)} \partial X^{2}=T_{x_{0}} \partial \Sigma_{f\left(t_{0}\right)} \times$ $\left(T_{x_{0}} \partial \Sigma_{f\left(t_{0}\right)}\right)^{\perp}$ : the copy of $T_{x_{0}} \partial \Sigma_{f\left(t_{0}\right)}$ and 2-planes containing vectors perpendicular to $T_{x_{0}} \partial \Sigma_{f\left(t_{0}\right)}$. Recall that by construction $\partial \Sigma_{f\left(t_{0}\right)}$ has nonpositive curvature, so 
the sectional curvature of $T_{x_{0}} \partial \Sigma_{f\left(t_{0}\right)}$ is nonpositive. On the other hand, consider that curve $\sigma:[0, \infty) \rightarrow \partial X^{2}$ defined by

$$
\sigma(t)=\left(\Phi_{f(t)}\left(\Pi\left(x_{0}\right)\right), t\right)
$$

and note that $\sigma\left(t_{0}\right)=\left(x_{0}, t_{0}\right)$ and $\sigma^{\prime}\left(t_{0}\right)$ is perpendicular to $T_{x_{0}} \partial \Sigma_{f\left(t_{0}\right)}$. Since $f^{\prime \prime}<0$, the acceleration vector $\sigma^{\prime \prime}$ points inwards; i.e. any tangent 2 -plane containing vectors perpendicular to $T_{x_{0}} \partial \Sigma_{f\left(t_{0}\right)}$ does not bend away from the interior. Therefore, we conclude that $(X, d)$ is of nonpositive curvature.

Recall that for every $t \geq 0, \partial \Sigma_{f(t)}$ contains a copy of $\Sigma$ and that $\phi$ is the identify map of $\Sigma$ which is a harmonic map by construction. Define $u_{t}: \Sigma \rightarrow X$ by setting $u_{t}(x)=(\phi(x), t)$. By property (i) and the fact that $\phi$ is harmonic, $u_{t}$ 's are energy minimizing maps for $t>0$. (In fact, this then shows that $u_{0}$ is also energy minimizing since the energies of $u_{0}$ and $u_{t}$ are equal.) By the discussion in property (iii) and by using condition (2) above, we can easily find points $x, y \in \Sigma$ so that $d\left(u_{t}(x), u_{t}(y)\right)>d\left(u_{s}(x), u_{s}(y)\right)$ for some $t, s$ with $t<s$.

REMARK 19. We will now show where the arguments of [Jo5] fail. First, we recall Jost's formulation of the energy functional. Let $(M, \mu)$ be a measure space, $(X, d)$ be an NPC space, and $h: M \times M \rightarrow \mathbf{R}$ be a nonnegative, symmetric function. The $h$-energy of $u: M \rightarrow X$ is

$$
E_{h}(u)=\iint h(x, y) d^{2}(u(x), u(y)) d \mu(x) d \mu(y) .
$$

Set

$$
h_{\epsilon}(x, y)=\chi_{B_{\epsilon}(x)}(y)
$$

where $\chi_{B_{\epsilon}(x)}$ is the characteristic function of the open ball $B_{\epsilon}(x)=\{z \in M$ : $\left.d_{M}(x, z)<\epsilon\right\}$. Let $\Gamma$ be a group acting on $M$ preserving $\mu$ and let $\mu_{\Gamma}$ be the induced measure on $M / \Gamma$. Let $\rho: \Gamma \rightarrow \operatorname{Isom}(X)$ be a isometric action of $\Gamma$ on $X$. Select a $\rho$-equivariant map $u_{0}: M \rightarrow X$ and set

$$
L_{\rho}^{2}(M, X)=\left\{v: M \rightarrow X \text { is } \rho \text { - equivariant with } d\left(v, u_{0}\right)<\infty\right\}
$$

where

The functional

$$
d^{2}(u, v)=\int d^{2}(u(x), v(x)) d \mu_{\Gamma}(x) .
$$

is defined by

$$
E_{h_{\epsilon}}: L_{\rho}^{2}(M, X) \rightarrow \mathbf{R}^{2} \cup \infty
$$

$$
E_{h_{\epsilon}}(u)=\iint h_{\epsilon}(x, y) d^{2}(u(x), u(y)) d \mu_{\Gamma}(x) d \mu_{\Gamma}(y) .
$$

The energy functional

$$
E: L_{\rho}^{2}(M, X) \rightarrow \mathbf{R}^{2} \cup \infty
$$

is defined as the $\Gamma$-limit (see [Jo5] for the definition) of normalized functionals $\left\{\frac{1}{\eta_{\epsilon}} E_{h_{\epsilon}}\right\}$ where

$$
\eta_{\epsilon}=\inf _{v \in L_{\rho}^{2}(M, X)} E_{h_{\epsilon}}(v) .
$$

To prove the uniqueness of energy minimizing map, [Jo5] employs the following argument. For $x, y \in M$,

$$
d^{2}\left(u_{t}(x), u_{t}(y)\right) \leq(1-t) d^{2}\left(u_{0}(x), u_{0}(y)\right)+t d^{2}\left(u_{1}(x), u_{1}(y)\right)
$$


by inequality (5). Thus

$$
\begin{aligned}
E_{h_{\epsilon}}\left(u_{t}\right) & =\iint h_{\epsilon}(x, y) d^{2}\left(u_{t}(x), u_{t}(y)\right) d \mu_{\Gamma}(x) d \mu_{\Gamma}(y) \\
& \leq \iint h_{\epsilon}(x, y)\left((1-t) d^{2}\left(u_{0}(x), u_{0}(y)\right)+t d^{2}\left(u_{1}(x), u_{1}(y)\right)\right) d \mu_{\Gamma}(x) d \mu_{\Gamma}(y) \\
& \leq(1-t) E_{h_{\epsilon}}\left(u_{0}\right)+t E_{h_{\epsilon}}\left(u_{1}\right) .
\end{aligned}
$$

If $u_{0}$ and $u_{1}$ are $h$-energy minimizers, then $u_{t}$ is also a $h$-energy minimizer and we must have equality. [Jo5] then concludes we must have equality in (9) for almost all $x, y \in M$. But line of argument is not correct since $h_{\epsilon}(x, y)=0$ if $d_{M}(x, y)>\epsilon$. Now we can try another symmetric function in place of $h_{\epsilon}$ which is everywhere positive, for example

$$
\tilde{h}_{\epsilon}(x, y)=e^{\frac{d_{M(x, y)}^{2}}{\epsilon}} .
$$

But we then run into another problem in that if $X$ is as in Example 17, $\tilde{h}_{\epsilon}$-energy minimizers do not exist, although we have shown that minimizers of the energy functional $E$ exist. The non-existence of the $\tilde{h}_{\epsilon}$-energy minimizing maps can be seen by noting (iii) which implies that $\tilde{h}_{\epsilon}$-energy of $v: M \rightarrow X, v(x)=\left(v_{1}(x), t(x)\right)$ is greater than that of $\bar{v}: M \rightarrow X$ defined by $\bar{v}(x)=\left(v_{1}(x), t(x)+\epsilon\right)$ for any $\epsilon>0$. As this example illustrates, maps which minimize the energy functional $E$ are not necessarily a limit of a sequence of minimizing maps corresponding to a sequence of approximating functionals of $E$. We note that Example 17 does not contradict the existence result for $\rho$-equivariant $h$-energy minimizing maps (Theorem 4.2.1 of [Jo5]) since $\rho$ as in Example 17 fixes an equivalence class of rays; namely $\rho$ fixes $[\gamma]$ where $\gamma:[0, t) \rightarrow X$ is defined by $\gamma(t)=(x, t)$ for a fixed $x \in \Sigma$.

\section{Uniqueness of harmonic maps between surfaces}

We now restrict our attention to maps between compact Riemann surfaces $\left(\Sigma_{1}, z\right),\left(\Sigma_{2}, w\right)$ of the same genus $g \geq 2$. Here, $z$ and $w$ are the local complex coordinates of $\Sigma_{1}$ and $\Sigma_{2}$ respectively. Consider a (possibly degenerate and nonsmooth) metric $\lambda|d w|^{2}$ on $\Sigma_{2}$ with $\lambda$ a local function satisfying $\triangle \log \lambda \geq 0$ weakly. We remark that such a metric induces a NPC distance function $d$ on $\Sigma_{2}$ (see [Me]). We have the following existence theorem for harmonic maps (see $[\mathbf{M e}]$ ):

THEOREM 20. Let $\phi: \Sigma_{1} \rightarrow \Sigma_{2}$ be a diffeomorphism. There exists a map $u$ : $\Sigma_{1} \rightarrow \Sigma_{2}$ homotopic to $\phi$ and energy minimizing with respect to $\lambda|d w|^{2}$. Moreover, $u$ can be constructed as a (pointwise) limit of diffeomorphism $u_{i}$ which are energy minimizing maps with respect to smooth Riemannian metrics on $\Sigma_{2}$ with nonpositive curvature.

Let $u: \Sigma_{1} \rightarrow \Sigma_{2}$ be an energy minimizing map. Its Hopf differential $\Phi$ is defined locally by $\varphi d z^{2}$ where

$$
\varphi=\pi\left(\frac{\partial}{\partial x}, \frac{\partial}{\partial x}\right)-\pi\left(\frac{\partial}{\partial y}, \frac{\partial}{\partial y}\right)-2 i \pi\left(\frac{\partial}{\partial x}, \frac{\partial}{\partial y}\right) .
$$

Even when the target of $u$ is nonsmooth, $\varphi$ is holomorphic and $\Phi$ is a holomorphic quadratic differential on the Riemann surface $\Sigma_{1}$ (see for example [Sc] where the proof of this property depends only on the domain variations). Since $\Phi$ is a holomorphic differential, it has finite zeroes counting multiplicities. For each $p \in \Sigma_{1}$, 
there exists a natural parameter $z$ with $z(p)=0$ so that $\Phi$ can be written as

$$
\Phi=\left(\frac{m+2}{2}\right)^{2} z^{m} d z^{2}
$$

in a neighborhood of $p$. Here, the integer $m$ is the vanishing order $\Phi$ at 0 . The integral curves of the distribution $\left\{v \in T \Sigma_{1}: \Phi(v, v) \leq 0\right\}$ is called the vertical trajectory. The Hopf differential gives a geometric picture of harmonic maps as the vertical trajectories give the direction of minimal stretch.

For any energy minimizing map $u: \Sigma_{1} \rightarrow \Sigma_{2}$ and a point $p \in \Sigma_{1}$, we define a tangent map $u_{*}$ at $p$ by blowing up the map $u$. For $p \in \Sigma_{1}$, we let $z$ be a natural parameter with $z(p)=0$. We define

$$
\begin{gathered}
E(\sigma)=\int_{\{|z|<\sigma\}}|\nabla u|^{2} d x d y, \\
I(\sigma)=\int_{\{|z|=\sigma\}} d^{2}(u(z), u(0)) d s
\end{gathered}
$$

and

$$
\operatorname{ord}(\sigma)=\frac{\sigma E(\sigma)}{I(\sigma)} .
$$

The function $\sigma \mapsto \operatorname{ord}(\sigma)$ is monotonically nondecreasing with $\tau=\lim _{\sigma \rightarrow 0} \operatorname{ord}(\sigma) \geq$ 1 and the function $\sigma \mapsto \sigma^{-2 \tau-1} I(\sigma)$ is nondecreasing (see [GS]). Consider the blow up maps $u_{\sigma}: D \rightarrow\left(\Sigma_{2}, d_{\sigma}\right)$ defined by $u_{\sigma}(z)=u(\sigma z)$. Here the distance function $d_{\sigma}$ on the set $\Sigma_{2}$ is defined by

$$
d_{\sigma}(p, q)=\frac{d(p, q)}{\sqrt{\frac{1}{\sigma} I(\sigma)}}
$$

For any sequence $\sigma_{i} \rightarrow 0$, there exists a subsequence $\left\{u_{\sigma_{i^{\prime}}}\right\}$ of $\left\{u_{\sigma_{i}}\right\}$ that converges to a homogeneous energy minimizing map $u_{*}: D \rightarrow\left(\mathbf{C}, d_{0}\right)$ (in the sense that the pull back distance functions $\delta_{i^{\prime}}(\cdot, \cdot)=d_{\sigma}\left(u_{\sigma_{i^{\prime}}}(\cdot), u_{\sigma_{i}^{\prime}}(\cdot)\right)$ converge to $d_{0}\left(u_{*}(\cdot), u_{*}(\cdot)\right)$ where $d_{0}$ is the distance function induced by a cone metric $\alpha^{2}|w|^{2(\alpha-1)}|d w|^{2}$ on the complex plane $\mathbf{C}$. (see $[\mathbf{M e}]$ for more details). We call $u_{*}$ a tangent map at $p$.

Tangent map $u_{*}$ at point $p \in \Sigma_{1}$ can be classified by the following lemma due to Kuwert (Lemma 3 of $[\mathbf{K u}]$ ).

Lemma 21. Let the reference map $w_{0}: \mathbf{C} \rightarrow \mathbf{C}$ be defined as follows:

$$
w_{0}(x)= \begin{cases}z & \text { for } k=0 \\ \left(\frac{1}{2}\left(k^{-\frac{1}{2}} z^{\frac{m+2}{2}}+k^{\frac{1}{2}} \bar{z}^{\frac{m+2}{2}}\right)\right) & \text { for } 0 \leq k \leq 1 .\end{cases}
$$

Assume that $u_{*}$ is normalized so that its Hopf differential is given in terms of the natural parameters. Then after a suitable rotation, one of the following alternatives holds:

(a) $u_{*}(z)=w_{0}(z)^{\frac{\tau}{\alpha}}$ where $k=0, \frac{\tau}{\alpha} \in \mathbf{N}$

(b) $u_{*}(z)=w_{0}(z)^{\frac{\tau}{\alpha}}$ where $0<k<1, \frac{\tau}{\alpha} \in \mathbf{N}$ and $m=2(\tau-1) \in \mathbf{N}$

(c) For $l \in\{1,2, \ldots, m+2\}$, there exists $\omega_{l} \in S^{1}$ such that $u_{*}\left(r e^{i \theta_{0}}\right)=\left|w_{0}\left(r e^{i \theta_{0}}\right)\right|^{\frac{\tau}{\alpha}} \omega_{l}$ for $\left(l-\frac{1}{2}\right) \frac{2 \pi}{m+2} \leq \theta_{0} \leq\left(l+\frac{1}{2}\right) \frac{2 \pi}{m+2}$ where $k=1, m=2(\tau-1) \in \mathbf{N}$ and $\angle\left(\omega_{l}, \omega_{l+1}\right) \geq$ $\frac{\pi}{\alpha}$. 
Furthermore, following the proof of Lemma 5 of $[\mathbf{K u}]$, we see that if $m<\infty$ where $m$ is the vanishing order of the Hopf differential $\Phi$ at $p$, then either $k=0$ or

$$
k=\mu-\sqrt{\mu-1}
$$

where

$$
\mu=\lim _{\sigma \rightarrow 0} \frac{1}{\pi \sigma^{m+3}} I(\sigma)=\lim _{\sigma \rightarrow 0} \frac{E(\sigma)}{\tau \pi \sigma^{m+2}} .
$$

In particular, we see that all tangent maps at a given point have the same stretch factor $k$. We will call $k: \Sigma_{1} \rightarrow[0,1]$ the stretch function of $u$.

The function $k$ gives the local behavior of the map $u$. More precisely, if $k(p)<1$ then $u^{-1}(u(p))$ is a single point and if $k(p)=1$, then $u^{-1}(u(p))$ is a union of vertical trajectories. Using this analysis, we now prove Theorem 4.

Proof of Theorem 4. Let $k_{0}$ and $k_{1}$ be the stretch functions of $u_{0}$ and $u_{1}$ respectively. By Corollary 13, equation (2), equation (10), we see that $k_{0} \equiv k_{1}$. Hence, $\left\{p: k_{0}(p)=1\right\}=\left\{p: k_{1}(p)=1\right\}$. For $q \in \Sigma_{2}$, let $\mathcal{P}_{q}=\left\{p: u_{0}(p)=q\right\}$. Without the loss of generality assume that $u_{0}$ is a limit of diffeomorphisms hence the set $\mathcal{P}_{q}$ is connected. We have that $k_{1}(p)=k_{0}(p)=1$ for all $p \in \mathcal{P}_{q}$, hence $u_{1}$ maps $\mathcal{P}_{q}$ to a single point. Therefore, the map $u_{1} \circ u_{0}^{-1}: \Sigma_{2} \rightarrow \Sigma_{1}$ is a well-defined map and is homotopic to the identity. Since genus of $\Sigma_{2}$ is $\geq 2, u_{1} \circ u_{2}$ must have a fixed point $q$. Thus, for $p \in u_{0}^{-1}(q), u_{0}(p)=q=u_{1}(p)$ and $d\left(u_{0}(p), u_{1}(p)\right)=0$. By Corollary $12, d\left(u_{0}, d_{1}\right) \equiv 0$ and therefore $u_{0} \equiv u_{1}$.

\section{Applications}

7.1. Preissman's Theorem. An immediate consequence of the uniqueness of equivariant harmonic maps is the Preissman's Theorem for compact metric space of curvature bounded from above by $\kappa<0$.

TheOREM 22 (Preissman's Theorem). Let $N$ be a compact metric space whose univeral cover $X$ is a metric space of curvature bounded from above by $\kappa<0$. Then every abelian subgroup of the fundamental group is infinite cyclic, i.e. isomorphic to $\mathbf{Z}$.

Proof. Let $A \subset \pi_{1}(N)$ be an abelian subgroup and $\alpha, \beta \in A$. The homotopy between $\alpha \beta$ and $\beta \alpha$ induces a map $g: T^{2} \rightarrow N$ from a two dimensional torus $T^{2}$. Let $a=g_{*}^{-1}(\alpha)$ and $b=g_{*}^{-1}(\beta)$. Let $u: T^{2} \rightarrow N$ be a harmonic map homotopic to $g$. The lift of $\tilde{u}: \mathbf{R}^{2} \rightarrow X$ is a $g_{*}$-equivariant harmonic map. Furthermore, $\alpha \circ \tilde{u}$ is $g_{*}$-equivariant (where we consider $\alpha: \mathbf{R}^{2} \rightarrow \mathbf{R}^{2}$ acting by deck transformation):

$$
\begin{aligned}
(\alpha \circ \tilde{u})(a x) & =\alpha(\tilde{u}(a x)) \\
& =\alpha\left(g_{*}(a) \tilde{u}(x)\right) \\
& =\alpha \circ \alpha \circ \tilde{u}(x) \\
& =g_{*}(a)(\alpha \circ \tilde{u})(x)
\end{aligned}
$$

and

$$
\begin{aligned}
(\alpha \circ \tilde{u})(b x) & =\alpha(\tilde{u}(b x)) \\
& =\alpha\left(g_{*}(b) \tilde{u}(x)\right) \\
& =\alpha \circ \beta \circ \tilde{u}(x) \\
& =\beta \circ \alpha \circ \tilde{u}(x) \\
& =g_{*}(b)(\alpha \circ \tilde{u})(x) .
\end{aligned}
$$


Hence, by Theorem 2, $\tilde{u}$ must maps into a geodesic. Thus, $u$ maps into a closed geodesic, say $\gamma$. Thus, $\tilde{u}_{*}(a)$ and $\tilde{u}_{*}(b)$ are multiples of $\gamma$ and this shows $\alpha$ and $\beta$ are both contained in a cyclic group generated by $\gamma$. This cyclic group must be infinite since if $\gamma^{k}$ is equal to the identity element for some $k \in \mathbf{N}$, then this contradicts the uniqueness of geodesics.

7.2. Necessary condition for the existence of equivariant harmonic maps. If $X$ is a simply connected manifold of nonpositive curvature and has no flat half-strip, F. Labourie $[\mathbf{L}]$ has shown that there exists a $\rho$-equivariant harmonic map if and only if $\rho(\Gamma)$ is a reductive subgroup of $\operatorname{Isom}(X)$. This generalized an earlier result of $\mathrm{K}$. Corlette $[\mathbf{C}]$ for equivariant harmonic maps into symmetric space of noncompact type. Furthermore, Korevaar and Schoen [KS3] and Jost [Jo2] has shown that the existence of $\rho$-equivariant harmonic map if $X$ is of finite rank and if $\rho(\Gamma) \subset \operatorname{Isom}(X)$ does not fix an equivalence class of geodesic rays. Using this result, we will prove the generalization of Labourie's theorem, Theorem 5 and Theorem 6 .

Recall that a geodesic ray is a arclength parameterization of a geodesic from the interval $[0, \infty)$ and a geodesic line is a arclength parameterization of a geodesic from the real line $\mathbf{R}$. Two geodesic rays $\gamma_{1}, \gamma_{2}$ (resp. geodesic lines $\bar{\gamma}_{1}, \bar{\gamma}_{2}$ ) are said to be equivalent if the Hausdorff distance between $\gamma_{1}([0, \infty))$ and $\gamma_{2}([0, \infty))$ (resp. $\gamma_{1}(\mathbf{R})$ and $\left.\gamma_{2}(\mathbf{R})\right)$ is finite.

Denote the set of equivalent geodesic rays by $\mathcal{R}$. If $X$ is a locally compact space, we set $\partial X=\mathcal{R}$ and there is a natural Hausdorff topology on $\bar{X}=X \cup \partial X$ so that $\bar{X}$ is the closure of $X$ and $\bar{X}$ is compact. Here, we are considering $X$ that is not necessarily locally compact, yet we still view $\mathcal{R}$ as points at infinity of $X$. It is well known that for every $P \in X$, there exists a unique representative geodesic ray $\sigma_{P}$ in each equivalent class $[\sigma] \in \mathcal{R}$ with initial point $P$. A subgroup $G$ of $\operatorname{Isom}(X)$ is said to have a infinite fixed point if there exists $[\sigma] \in \mathcal{R}$ so that for every $\gamma \in[\sigma]$ and $\phi \in G, \phi \circ \gamma \in[\sigma]$.

Suppose $G \subset \operatorname{Isom}(X)$ fixes $[\sigma] \in \mathcal{R}$. We define the a map which pushes points in $X$ toward the direcion of $[\sigma]$. For $P \in X$, let $\sigma_{P}:[0, \infty) \rightarrow X$ the geodesic ray with $\gamma_{P}(0)=P$. Define $\Phi_{[\sigma], \epsilon}: X \rightarrow X$ by

$$
\Phi_{[\sigma], \epsilon}(P)=\sigma_{P}(\epsilon)
$$

We show:

LEMma 23. The map $\Phi_{[\sigma], \epsilon}$ is distance decreasing.

Proof. Suppose $d\left(\Phi_{[\sigma], \epsilon}(P), \Phi_{[\sigma], \epsilon}(Q)\right)=d(P, Q)+\delta$ for some $P, Q \in X$ and $\delta>0$. Then by the quadrilateral comparison,

$$
\begin{aligned}
d^{2}\left(\sigma_{P}(\epsilon), \sigma_{Q}(\epsilon)\right) & \leq\left(1-\frac{\epsilon}{t}\right) d^{2}(P, Q)+\frac{\epsilon}{t} d^{2}\left(\sigma_{P}(t), \sigma_{Q}(t)\right) \\
& =\left(1-\frac{\epsilon}{t}\right)\left(\sigma_{P}(\epsilon), \sigma_{Q}(\epsilon)-\delta\right)+\frac{\epsilon}{t} d^{2}\left(\sigma_{P}(t), \sigma_{Q}(t)\right)
\end{aligned}
$$

Let $t \rightarrow \infty$ to obtain

$$
d^{2}\left(\sigma_{P}(\epsilon), \sigma_{Q}(\epsilon)\right) \leq d^{2}\left(\sigma_{P}(\epsilon), \sigma_{Q}(\epsilon)\right)-\delta
$$

which is a contradiction.

Lemma 24. Let $[\sigma] \in \mathcal{R}$ and $I: X \rightarrow X \in G$. then $\Phi_{[\sigma], \epsilon}(I(P))=I \circ \Phi_{[\sigma], \epsilon}(P)$. 
Proof. Let $Q=I(P)$. Then $I\left(\sigma_{P}\right)$ is a geodesic ray in the equivalence class of $\sigma$ starting at $Q$ and $I \circ \Phi_{[\sigma], \epsilon}(P)=I\left(\sigma_{P}(\epsilon)\right)$ is a point on $I\left(\sigma_{P}\right)$ at a distance $\epsilon$ from $Q$. Furthermore, $\Phi_{[\sigma], \epsilon}(I(P))=\sigma_{I(P)}(\epsilon)=\sigma_{Q}(\epsilon)$ is a point on the geodesic ray in the equivalence class of $\sigma$ starting at $Q$ at a distance $\epsilon$ from $Q$. By uniqueness of the representation of $\sigma$ which starts at the point $Q$, we have $\Phi_{[\sigma], \epsilon}(I(P))=$ $I \circ \Phi_{[\sigma], \epsilon}(P)$.

Proposition 25. Let $M$ be a compact Riemannian manifold with fundamental group $\Gamma, X$ be a complete metric space of nonpositive curvature and $\rho: \Gamma \rightarrow$ $\operatorname{Isom}(X)$ be a homomorphism. Assume $G=\rho(\Gamma)$ fixes $[\sigma] \in \mathcal{R}$. If $u: M \rightarrow X$ is a $\rho$-equivariant harmonic map, then $\Phi_{[\sigma], t} \circ u$ is a harmonic map for all $t$.

Proof. By Lemma $23, d\left(\Phi_{[\sigma], t} \circ u(x), \Phi_{[\sigma], t} \circ u(y)\right) \leq d(u(x), u(y))$ for any $x, y \in X$. Therefore, $E(u)=E\left(\Phi_{[\sigma], t} \circ u\right)$. Furthermore, for $\gamma \in \Gamma, \Phi_{[\sigma], t}(u(\gamma x))=$ $\Phi_{[\sigma], t}(\rho(\gamma) u(x))=\rho(\gamma) \circ \Phi_{[\sigma], t}(u(x))$ by Lemma 24 and thus $\Phi_{[\sigma], t} \circ u$ is $\rho$-equivariant.

Now Theorem 5 is an easy consequence of the uniqueness theorem: Suppose $\rho$ fixes $[\sigma] \in \mathcal{R}$ and $u: \tilde{M} \rightarrow X$ is a $\rho$-equivariant harmonic map. Then since $\Phi_{[\sigma], \epsilon}: X \rightarrow X$ is distance decreasing, the energy of the map $u$ is equal to the energy of the map $\Phi_{[\sigma], \epsilon} \circ u$. By the uniqueness of $\rho$-equivariant harmonic map into a CAT $(-1)$ space, $u$ maps onto a geodesic in $X$.

More generally, we prove Theorem 6 . We first need to clarify the terminology used in the statement of that theorem. If $X$ is a smooth Riemannian manifold, then $X$ is said to have no flat half-strip if any Jacobi field along a geodesic line which has constant norm for every $t \geq t_{0}$ has constant norm for any $t$. For example, negatively curved manifolds and analytic manifolds possess this property. In absense of smooth structures (hence Jacobi fields), we will make the following definition of the condition of no flat half-strip.

Definition 26. A complete metric space of nonpositive curvature $X$ is said to have no flat half-strip if the following condition is satisfied: if $F:\left[t_{0}, \infty\right) \times\left[0, \epsilon_{0}\right] \rightarrow$ $X$ has the property that $t \mapsto F(t, \epsilon)$ is a geodesic for all $\epsilon \in\left[0, \epsilon_{0}\right]$ and the lengths of the curves $\epsilon \mapsto F(t, \epsilon), 0 \leq \epsilon \leq \epsilon_{0}^{\prime}$, has constant length for all $t \in\left[t_{0}, \infty\right)$ and all $\epsilon_{0}^{\prime} \in\left[0, \epsilon_{0}\right]$, then there exists a map $\tilde{F}: \mathbf{R} \times\left[0, \epsilon_{1}\right] \rightarrow X$ with $\epsilon_{1} \leq \epsilon_{0}$ so that $\tilde{F}(t, \epsilon) \equiv F(t, \epsilon)$ for $t \in\left[t_{0}, \infty\right)$ and $\epsilon \in\left[0, \epsilon_{1}^{\prime}\right], t \mapsto \tilde{F}(t, \epsilon)$ is a geodesic for all $\epsilon$ and the lengths for the curves $\epsilon \mapsto \tilde{F}(t, \epsilon), 0 \leq \epsilon \leq \epsilon_{1}^{\prime}$, has constant length of all $t \in \mathbf{R}$ and $\epsilon_{1}^{\prime} \in\left[0, \epsilon_{1}\right]$.

If $X$ is a smooth Riemannian manifold, then $F_{*}\left(\frac{\partial}{\partial \epsilon}\right)$ on the geodesic $t \rightarrow F(t, 0)$ is a Jacobi field, and coveresely, all Jacobi field can be obained in this way. Hence Definition 26 is equivalent to the definition of the no flat half strip property defined in terms of Jacobi fields.

Definition 27. A subgroup $G$ of $\operatorname{Isom}(X)$ is said to be reductive if there exists a closed convex set $X_{c}$ of $X$ globally fixed by $G$ so that $X_{c}$ is isometric to $X_{c}^{0} \times \mathbf{R}^{n}$ for some subset $X_{c}^{0}$ of $X_{c}, G=G_{1} \times G_{2}$ where $G_{1} \subset \operatorname{Isom}\left(X_{c}^{0}\right)$ and $G_{2} \subset \operatorname{Isom}\left(\mathbf{R}^{n}\right)$ and $G_{1}$ no infinite fixed point.

Suppose $G=\rho(\Gamma)$ has an infinite fixed point $[\sigma] \in \mathcal{R}$. Let $u_{t}=\Phi_{[\sigma], t} \circ u$ for $t \in[0, \infty)$. By Proposition 25, $u_{t}: \tilde{M} \rightarrow X$ is a one-parameter family of harmonic maps. 
For $x, y \in \tilde{M}$, let $\epsilon_{0}=d_{M}(x, y)$ and let $\sigma:\left[0, \epsilon_{0}\right] \rightarrow M$ be the geodesic between $x$ and $y$. Let $F:[0, \infty) \times\left[0, \epsilon_{0}\right] \rightarrow X$ be defined by $F(t, \epsilon)=u_{t}(\sigma(\epsilon))$. By construction $t \mapsto F(t, \epsilon)$ is a geodesic ray for all $\epsilon \in\left[0, \epsilon_{0}\right]$ and length of the curves $\epsilon \mapsto F(t, \epsilon), 0 \leq \epsilon \leq \epsilon_{0}$, is constant by Corollary 13. By the no half-strip property, $F$ extend to a map $\tilde{F}$ as in Definition 26 .

Let $X_{c}$ be the images of all geodesic lines asymptotic to $\gamma(t)=\tilde{F}(t, 0)$. The Product Decomposition Theorem (2.14) of $[\mathbf{B H}]$ says that $X_{c}$ is a convex subspace of $X$. Furthermore, if $\Pi$ is a restriction to $X_{c}$ of the projection from $X$ to $\bar{\gamma}_{x}(\mathbf{R})$, then $X_{c}^{0}=\Pi^{-1}\left(\bar{\gamma}_{x}(0)\right)$ is convex and $X_{c}$ is canonically isometric to the product $X_{c}^{0} \times \mathbf{R}$. Since being asymptotic is an equivalence relationship on the set of geodesic lines, $u(\tilde{M}) \subset X_{c}$. The map $v=\Pi \circ u$ is a harmonic map since a projection map onto a convex set is distance decreasing in an nonpositively curved space.

For $p \in X_{c}^{0}$, let $\bar{\sigma}_{p}: \mathbf{R} \rightarrow X_{c}^{0} \times \mathbf{R}$ be the geodesic line asymptotic to $\bar{\gamma}_{x}$ (where $X_{c}$ is identified with $\left.X_{c}^{0} \times \mathbf{R}\right)$ and so that $\bar{\sigma}_{p}(0)=p$. Thus, $\bar{\sigma}_{p}(t)=(p, t) \in X_{c}^{0} \times \mathbf{R}$. For $g \in G=\rho(\Gamma), g \circ \bar{\sigma}_{p}$ is a geodesic line asymptotic to $\bar{\gamma}_{x}$ and hence $g \circ \bar{\sigma}_{p}(\mathbf{R}) \subset$ $X_{c}=X_{c}^{0} \times \mathbf{R}$ and, for some $q \in X_{c}^{0}$, we can write

$$
g \circ \bar{\sigma}_{p}(t)=\left(q, t^{\prime}\right)=\bar{\sigma}_{q}\left(t^{\prime}\right) .
$$

Therefore we can decompose an element $g \in G$, by $g=\left(g_{1}, g_{2}\right)$ where $g_{1}(p)=q$ and $g_{2}(t)=t^{\prime}$. In this way, $G=G_{1}^{\prime} \times G_{2}^{\prime}$ where $G_{1}^{\prime} \subset \operatorname{Isom}\left(X_{c}^{0}\right)$ and $G_{2}^{\prime} \subset \operatorname{Isom}(\mathbf{R})$.

Moreover, if $G_{1}^{\prime}$ fixes an equivalence class of geodesic rays of $X_{c}^{0}$, then we can decompose $G_{1}^{\prime}$ in an analogous way as above using the harmonic map $v$. Thus, we can continue inductively until $G=G_{1} \times G_{2}$ and $X_{c}=X^{0} \times \mathbf{R}^{n}$ as in the statement of the theorem. This shows the necessary condition for the existence of a $\rho$-equivalent harmonic map. For the proof of the sufficient condition, we refer to [KS3] or [Jo2].

\section{References}

[ABB] S.B. Alexander, I.D. Berg, and R. L. Bishop. Geometric curvature bounds in Riemannian manifolds with boundary. Trans. AMS 339 (1993) 703-716.

[Al] S.I. Al'ber. On n-dimensional problems of the calculus of variations in the large. Sov. Math. Dokl. 6 (1964) 700-704.

[BH] M. Bridson and A. Haefliger. Metric Spaces of Non-Positive Curvature. Springer-Verlag, Berlin Heidelberg 1999.

[C] K. Corlette. Flat G-bundles with canonical metrics. J. Diff. Geom. 28 (1988) 361-382.

[EF] J. Eells and B. Fuglede. Harmonic maps between Riemannian polyhedra. Cambridge Tracts in Mathematics 142, Cambridge University Press, Cambridge 2001.

[ES] J. Eells and J.H. Sampson. Harmonic mappings of Riemannian manifolds. Amer. J. Math. 86 (1964) 109-160.

[CH] J.M. Coron and F. Hélein. Harmonic diffeomorphisms, minimizing harmonic maps, and rotational symmetry. Compositio Math. 69 (1989) 175-228.

[G] G. Gregori. Sobolev spaces and harmonic maps between sigular spaces. Calc. Var. 7 (1998) $1-18$.

[GS] M. Gromov and R. Schoen. Harmonic maps into singular spaces and p-adic superrigidity for lattices in groups of rank one. IHES Publ. Math. 76 (1992) 165-246.

[Hm] R. Hamilton. Harmonic maps of manifolds with boundary. Lecture notes 471. Sprinter, 1975.

[Hr] P. Hartman. On homotopic harmonic maps. Can. J. Math. 19 (1967) 673-687.

[Jo1] J. Jost. Equilibrium maps between metric spaces. Calc. Var. 2 (1994) 173-204.

[Jo2] J. Jost. Convex functionals and generalized harmonic maps of spaces of nonpositive curvature. Comm. Math. Helv. 70 (1995) 659-673. 
[Jo3] J. Jost. Generalized harmonic maps between metric spaces. Geom Anal. and Calc. Var. (1996) 143-174.

[Jo4] J. Jost. Generalized Dirichlet forms and harmonic maps. Calc. Var. 5 (1997) 1-19.

[Jo5] J. Jost. Nonpositive curvature: geometric and anlaytic aspects. ETC Lecture Notes, Birkhaäuser, Berlin 1997.

[JS] J. Jost and R. Schoen. On the existence of harmonic diffeomorphisms between surfaces. Invent. Math. 66 (1983) 353-359.

[KS1] N. Korevaar and R. Schoen. Sobolev spaces and harmonic maps for metric space targets. Comm. Anal. Geom. 1 (1993) 561-659.

[KS2] N. Korevaar and R. Schoen. Global existence theorem for harmonic maps to non-locally compact spaces. Comm. Anal. Geom. 5 (1997) 333-387.

[KS3] N. Korevaar and R. Schoen. Global existence theorems for harmonic maps: finite rank spaces and an approach to rigidity for smooth actions. Preprint.

[Ku] E. Kuwert. Harmonic maps between flat surfaces with conical singularities. Math. Z. 221 (1996) 421-430.

[L] F. Labourie. Existence d'applications harmoniques tordues a valeurs dans les variétés a courbure négative. Proc. Amer. Math. Soc. 111 (1991) 877-883.

[Me] C. Mese. Harmonic maps into spaces with an upper curvature bound in the sense of Alexandrov. to appear Math. Z.

[Re] Y.G. Reshetnyak. Nonexpanding maps in a space of curvature no greater than $K$. Siberian Math. Journ. 9 (1968) 918-927.

[Sa] J.H. Sampson. Some Properties and Applications of harmonic mapings. Ann. Scient. Ec. Norm. Sup. 11 (1977) 211-228.

[Se1] T. Serbinowski. Boundary regularity of harmonic maps to nonpositively curved metric spaces. Comm. Anal. Geom. 2 (1994) 139-154.

[Se2] T. Serbinowski. Harmonic maps into metric spaces with curvature bounded above. Ph.D. thesis, University of Utah, 1995.

[Sc] R. Schoen. Analytic aspects of the harmonic map problem. Seminar in non-linear partial differential equations. MSRI vol 2 (S.S. Chern, editor). Springer, Heidelberg and New York, 1985 .

[SY] R. Schoen and S-T. Yau. Existence of incompressible minimal surfaces and the topology of three dimensional manifolds with non-negative scalar curvature. Ann. of Math. 110 (1979) $127-142$. 